\title{
Interference among modulators
}

\author{
ROBERT A. RESCORLA \\ University of Pennsylvania, Philadelphia, Pennsylvania
}

\begin{abstract}
The effects of concurrent facilitation $(\mathrm{A}-, \mathrm{AX}+)$ and inhibition $(\mathrm{B}+, \mathrm{BY}-)$ training were studied in four autoshaping experiments with pigeons. The development and transfer of each type of modulation was slowed by concurrent training in the alternative paradigm. The interfering effect of facilitation on the development of inhibition contrasts with the positive effect of excitatory training. Implications for accounts of modulation are discussed.
\end{abstract}

There is a good deal of recent evidence that some Pavlovian procedures result in stimuli that serve a modulatory function. A simple conditioned stimulus (CS) may come to evoke a conditioned response (CR) because it signals the occurrence of an unconditioned stimulus (US). But under some circumstances, another stimulus may serve as a modulator of the functioning of that CS.

The two most commonly studied examples are facilitation (positive occasion-setting) and inhibition (negative occasion-setting). In the former, one stimulus, A, signals the US, but only in the presence of the modulator, X. Extensive studies done by Holland $(1983,1985)$ and Rescorla (1985) suggest that A will develop a direct association with the US, but that X will serve to facilitate or set the occasion for A's evoking its response. Similarly, in inhibitory paradigms, A signals the US, except in the presence of the modulator X. Under those circumstances, A becomes associated with the US, but the presence of $X$ interferes with A's evoking its response. One interpretation of such findings is that $\mathrm{X}$ modulates the response to $\mathrm{A}$.

The two paradigms are procedurally opposites of each other. Moreover, considerable evidence is consistent with the conclusion that they result in functionally opposite learning. For instance, an $X$ trained as a facilitator and a $Y$ trained as an inhibitor will have opposite effects on the performance to a target CS. Moreover, they will counteract each other's modulatory influence on that CS if they are presented in combination (see, e.g., Rescorla, 1987). Similarly, the two training procedures, if administered sequentially to the same stimulus will interfere with each other in learning. Facilitation training serves as an extinction procedure for inhibition, and vice versa (Rescorla, 1986, 1987).

The present experiments investigated the effects of administering the two training paradigms to the same stimulus concurrently. In Experiments 1, 3, and 4 several stimuli were trained as inhibitors, some of which were

This research was supported by National Science Foundation Grants BNS 83-08176 and BNS-88-03514. This work benefited from discussions with Dale Swartzentruber. Correspondence concerning this article should be addressed to R. A. Rescorla, Department of Psychology, University of Pennsylvania, 3815 Walnut Street, Philadelphia, PA, 19104. also trained as facilitators. In Experiments 2 and 4, several stimuli were trained as facilitators, some of which were also trained as inhibitors. The question of interest was the degree to which such additional training would promote or hinder the primary modulatory learning.

There are good reasons to anticipate any of three results of such experiments. First, the reasoning described above would lead one to anticipate an interfering effect of concurrent training. Training as a facilitator should interfere with the effects of training as an inhibitor, and vice versa. Second, recent evidence from intermixing simple excitatory training of a stimulus might lead one to anticipate an enhancing effect. Such excitatory training has a substantial augmenting effect on the development of modulators (see, e.g., Rescorla, 1991). Because that augmentation seems to occur for a wide range of modulators, excitatory training has been interpreted as augmenting the salience of the stimulus, making it more susceptible to modulatory learning. One might also expect any modulatory training to enhance the salience of a stimulus; therefore, it seems possible that such training would also enhance other modulatory training. That is, concurrent facilitory training might enhance inhibitory learning, and vice versa, because of its enhancing effect on the salience of the stimulus. Finally, the intermixing of the training of $\mathrm{X}$ in both a facilitory $\mathrm{A}-, \mathrm{XA}+$ paradigm and an inhibitory $\mathrm{B}+, \mathrm{XB}$ - paradigm constitutes an "ambiguous cue" training procedure for stimulus $\mathrm{X}$. The $\mathrm{X}$ signals the reinforcement of one stimulus, $A$, but the nonreinforcement of another stimulus, B. Recent instrumental training experiments done by Holland (1991) have found such a paradigm to endow $\mathrm{X}$ with both modulatory effects. Indeed, the ability of $X$ to facilitate $A$ and to inhibit $B$ apparently developed without interference. It is therefore of interest to investigate such procedures in a Pavlovian paradigm, such as autoshaping, in which much of the other modulatory work has been conducted.

All of the present experiments used autoshaping procedures in which pigeon subjects received food following the brief illumination of a keylight and in which conditioning was assessed by the rate of pecking at that keylight. In each case, the modulatory effect of $X$ was assessed by its ability to enhance or depress responding to $A$. 


\section{EXPERIMENT 1}

In this experiment, each of two diffuse stimuli (a white noise and a houselight) was trained as a conditioned inhibitor based on a localized keylight excitor. Concurrently, one of those diffuse stimuli was also trained as a facilitator for another keylight. The question of interest was the rate of development of inhibition as a function of whether or not the stimulus was undergoing concurrent facilitation training.

\section{Method}

Subjects and Apparatus. The subjects were 16 female Carneau pigeons about 1 year old. They had participated in another autoshaping experiment that had different stimuli and a different response key. The assignment of birds to groups in the present experiment was random with respect to their previous treatments. They were housed in pairs and maintained at $80 \%$ of their free-feeding weights.

The apparatus consisted of eight identical operant chambers, each measuring $27 \times 27 \times 35 \mathrm{~cm}$. The metal front panel of each chamber had a $5 \times 5 \mathrm{~cm}$ food magazine in its center, located $5 \mathrm{~cm}$ above the wire mesh floor. A $11.8 \times 14.5 \mathrm{~cm}$ response key, constructed of clear Lucite acrylic, was centered $9 \mathrm{~cm}$ above the magazine, behind a $10 \times 8 \mathrm{~cm}$ rectangular opening in the chamber wall. Located $.2 \mathrm{~cm}$ behind the response key was a Magnavox (Model CK3923) color television. This was connected to a computer programmed to generate and display visual stimuli. A black opaque strip blocked the top half of the screen from view in four of the boxes and the bottom half of the screen in the other four boxes. Four stimuli, white circular disks that were $2.2 \mathrm{~cm}$ in diameter and contained various patterns, could be presented in the center of the visible portion of the screen. One pattern consisted of $1-\mathrm{mm}$ thick parallel black lines spaced $1 \mathrm{~mm}$ apart on the white background and slanted $45^{\circ}$ from the vertical. A second pattern consisted of .5 -mm-thick parallel black lines spaced $3 \mathrm{~mm}$ apart and slanted $135^{\circ}$ from the vertical. A third pattern consisted of a set of eight evenly spaced black wedge-shaped sectors arrayed around the center of the disk, generating a pinwheel-like pattern. The fourth stimulus was the simple white disk without any pattern.

The other three walls and ceiling of the chambers were composed of clear Plexiglas. These chambers were placed in sound-and lightattenuating shells with ventilation fans that provided background noise of $62 \mathrm{~dB}$ re $20 \mu \mathrm{N} / \mathrm{m}$. On the rear wall of the shells was mounted a speaker that permitted the presentation of an 80-dB white noise. Also on this wall was a 6-W bulb that was continuously illuminated during the session, except during the operation of the food hopper. The hopper contained Purina Pigeon Grain. The bulb could also be interrupted at a rate of $1 / \mathrm{sec}$ to provide a diffuse visual stimulus.

Experimental events were automatically controlled by relay equipment and microprocessors located in an adjoining room.

Procedure. Because of the birds' past experience in an autoshaping experiment, no magazine training was necessary. On each of the first 20 days, the birds received conditioned inhibition training. In those sessions, a 5-sec presentation of the $45^{\circ}$ pattern (A) was the excitor and 15-sec presentations of the white noise (N) and interruption of the houselight (L) served as the inhibitors. Each session contained 12 presentations of $\mathrm{A}$ alone followed by $5 \mathrm{sec}$ of food and 12 presentations each of $A$ accompanied by $L$ and by $N$, but not followed by food. On the compound trials, the 15-sec diffuse stimulus was initiated $10 \mathrm{sec}$ prior to the onset of the keylight.

Intermixed with this training, half the animals received facilitation training with $\mathrm{N}$ and half received facilitation training with $\mathrm{L}$. For this purpose, each session also contained 24 presentations of the $135^{\circ}$ pattern (B). On 12 of the trials, B was presented without reinforcement; on the remaining 12 , it was accompanied by the diffuse stimulus and it terminated in $\mathbf{5 ~ s e c}$ of grain. The intertrial interval
(ITI) in this and all subsequent phases was variable around a mean of $1 \mathrm{~min}$.

Over the next 3 days, the 5-sec pinwheel pattern was given training intended to make it a transfer target for the diffuse modulators. On the 1st day, it was presented 36 times, each ending in $5 \mathrm{sec}$ of grain. On the next 2 days, it was presented in the same manner but without grain. This training and extinction treatment has been used routinely to generate targets that are sensitive to modulation (see, e.g., Rescorla, 1987). The next day contained a retraining session with the modulators, identical to those used above.

On the next day, the animals received a test session. The first half of the test consisted of half a session of modulation training. This was followed without interruption by two reinforced presentations of the pinwheel, and then by six nonreinforced presentations each of the pinwheel alone and accompanied by $\mathrm{L}$ and by $\mathrm{N}$. This test was intended to assess the inhibitory modulatory power of $L$ and $\mathrm{N}$ on a target stimulus with which neither had received training.

\section{Results and Discussion}

Figure 1 shows the results of the modulatory training sessions. The mean response rate is shown during each stimulus combination, in blocks of two sessions. The solid symbols show responding during the excitatory A when it was presented alone and in combination with the diffuse inhibitory modulators. Because there were no substantial differences between $\mathrm{L}$ and $\mathrm{N}$, the data have been combined across their identities but separated according to whether the inhibitor had otherwise received facilitory $(\mathrm{X})$ training or not $(\mathrm{Y})$.

It is clear that responding to A alone developed rapidly and came to be inhibited in the presence of both diffuse stimuli. However, this inhibition developed more rapidly and remained asymptotically more substantial for the diffuse stimulus not otherwise given facilitation training $(Y)$. On the final 2 days of training, both inhibitors suppressed responding to $\mathrm{A}$ [Wilcoxon $T \mathrm{~s}(15)=0, p s<.01]$. But the suppression was less substantial for the facilitory inhibitor; responding to YA was less than that to XA $[T(15)=$ $17, p<.02]$.

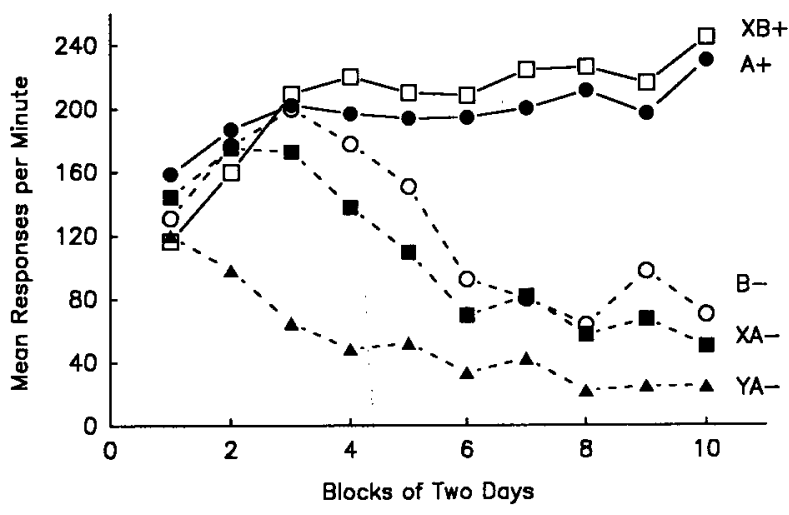

Figure 1. Acquisition of inhibition in Experiment 1. The filled symbols show responding during the excitatory keylight $A$ when it was presented alone and when it was in compound with the diffuse inhibitory $X$ and $Y$ stimuli. The open symbols show responding to $B$ when it was presented alone and when it was accompanied by $X$. The + and - indicate reinforcement and nonreinforcement, respectively. 


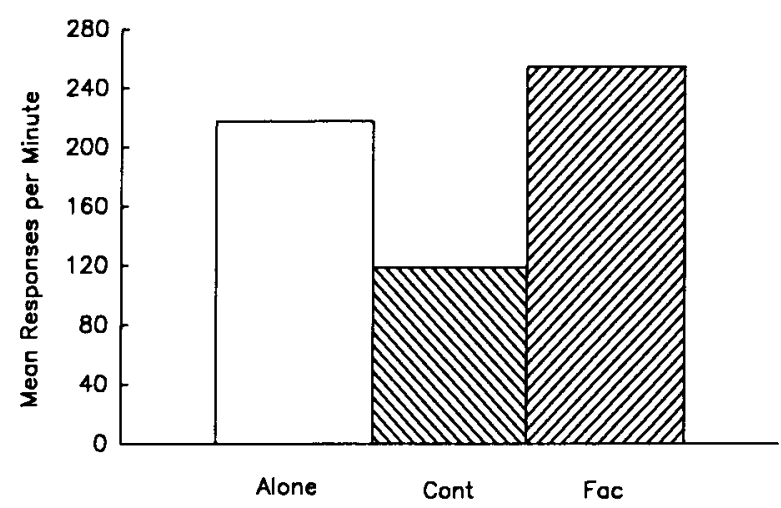

Figure 2. Transfer of inhitition in Experiment 1. Responding is shown to a keylight transfer stimulus when it was presented alone and when it was in combination with the facilatory and control inhibitor.

The open symbols show responding to the B stimulus when it was presented alone and in the presence of the facilitory inhibitor. That diffuse stimulus rapidly came to promote responding to the $\mathrm{B}$ stimulus. For instance, on the final block of training, responding to $B$ was substantially greater in the presence of $\mathrm{X}$ than in its absence $[T(15)=3, p<.01]$.

Figure 2 shows the results of the transfer test with the pinwheel stimulus, which evoked substantial responding when presented alone. However, this responding was depressed in the presence of the control inhibitor $[T(15)=$ $12, p<.01$, but it was augmented in the presence of the facilitory inhibitor $[T(15)=12, p<.01]$.

These results suggest that concurrent facilitation training with another target interferes with the development of inhibition. This interference was evident both in the slowed and less complete development of modulation of the original target and in the reduced transfer of inhibition to a novel target. Indeed, although the present experiment lacked appropriate controls, it suggests that the facilitation training was the more potent of the two procedures. The X stimulus trained both as a facilitator and inhibitor promoted, rather than depressed, responding to the transfer target.

\section{EXPERIMENT 2}

This experiment examined an issue complementary to that considered in Experiment 1-the effect of concurrent inhibitory training on the development of facilitation. Two different diffuse stimuli received training as facilitators, but one of these stimuli was also trained as an inhibitor with a different target. Subsequently, transfer of the diffuse stimuli to a novel target was assessed.

Although the details of this experiment were in most respects like those of Experiment 1, one procedural change was necessitated by the manner in which facilitation learning frequently expresses itself in this preparation. Differences in the amount of facilitation controlled by $X$ and $Y$ as a result of $A-, X A+$ and $B-, Y B+$ training are often evident primarily in the different levels of responding to $\mathrm{A}$ and $\mathrm{B}$ when they are presented alone. Responding to the XA and YB compounds will often be at very high and comparable levels despite $\mathrm{X}$ and $\mathrm{Y}$ 's being differentially strong as facilitators. That has two implications. First, it is not informative to train $X$ and $Y$ as facilitators of the same target, since it is unlikely that differences could be observed in responding to the compounds over the course of such training. Second, to assess accurately the degree of facilitation, one must transfer both stimuli to a common target. Consequently, in this experiment, the two facilitators were trained with different target keylights and a transfer test was administered.

\section{Method}

Subjects and Apparatus. The subjects were 16 experienced female pigeons with histories similar to those of the pigeons in Experiment 1 and maintained in a similar fashion. The apparatus was that from Experiment 1.

Procedure. On the first 14 days of the experiment, all animals received concurrent facilitation training with the diffuse noise and light. For half the animals, the $45^{\circ}$ stimulus served as the target keylight for the noise and the $135^{\circ}$ stimulus served as the target keylight for the light. For the other half of the animals, the stimuli were interchanged. The keylights were all $5 \mathrm{sec}$ long, the diffuse stimuli were $15 \mathrm{sec}$ long, and on compound trials the diffuse stimuli began $10 \mathrm{sec}$ prior to the keylight onset. Each session contained 12 reinforced presentations of each of the two compounds and 12 nonreinforced presentations of each of the keylight elements alone. In addition, each animal received 12 reinforced presentations of the pinwheel presented alone and 12 nonreinforced presentations of the pinwheel accompanied by either $\mathrm{L}$ or $\mathrm{N}$. For half the animals, $\mathrm{L}$ was treated as an inhibitor for the pinwheel whereas for the other half $\mathrm{N}$ received that treatment.

On the next 5 days, all animals received training of the white circle (W) as a target for transfer. On each of the first 2 days of this phase, the animals received 36 reinforced presentations of $W$. On the next 3 days, they received 36 nonreinforced presentations of $\mathrm{W}$. On the following day, all animals received a single additional modulation training session.

The next day contained a test session. That test began with a half session of modulation training, continued without interruption with four nonreinforced presentations of $W$, and then with six presentations each of $\mathrm{W}$ alone and accompanied by $\mathrm{L}$ and $\mathrm{N}$.

\section{Results and Discussion}

Figure 3 shows the course of responding during modulation training. The solid symbols display the results on the facilitation training trials, separated for the two modulators (squares, $\mathrm{A}-/ \mathrm{XA}+$, and circles, $\mathrm{B}-/ \mathrm{YB}+$ ) according to whether the stimuli were reinforced (solid lines) or not (dashed lines). The open symbols show responding on the inhibitory trials with one of those facilitators, $\mathrm{C}+/ \mathrm{XC}-$.

It is clear that facilitation developed rapidly to both modulators, with responding becoming substantially greater during the XA and YB facilitator/target compounds than during the $A$ and $B$ targets alone. As anticipated, the facilitators proved differentially effective, but this was displayed in terms of differences in responding to the targets alone. By the final day of training, both compounds showed greater responding than did their targets presented separately $[T \mathrm{~s}(15)=0, p s<.01]$. However, 


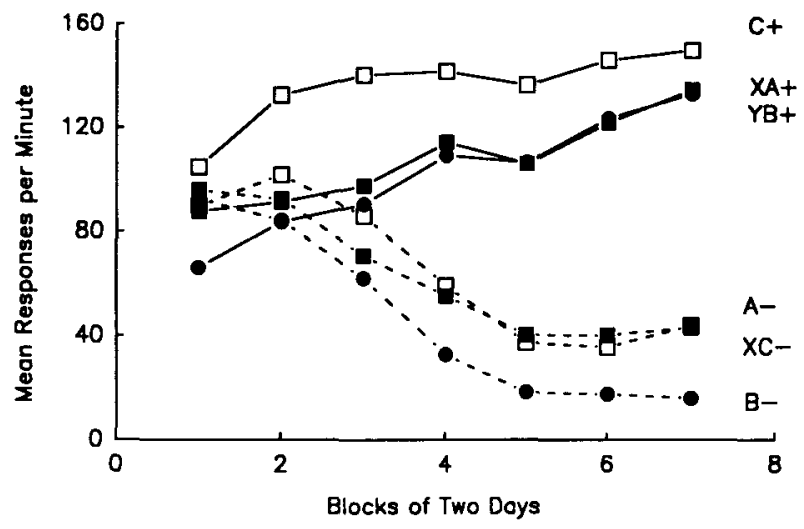

Figure 3. Acquisition of facilitation in Experiment 2. The filled symbols show responding during the $A$ and $B$ keylights when they were presented alone and when they were in combination with the diffuse facilitators $X$ and $Y$. The open symbols show responding to $C$ when it was presented alone and when it was accompanied by $X$. The + and - indicate reinforcement and nonreinforcement, respectively.

the control facilitator, $Y$, was stronger as assessed by the greater difference between $\mathrm{YB}$ and $\mathrm{B}$ than between $\mathrm{XA}$ and $A[T(15)=14, p<.01]$.

Responding to the diffuse $\mathrm{X}$ stimulus on its inhibitory training trials is shown in the open symbols. It is clear that this stimulus rapidly became capable of inhibiting the $\mathrm{C}$ target. On the final block of training, responding was reliably lower to $\mathrm{XC}$ than to $\mathrm{C}$ alone $[T(15)=3, p<.01]$.

Figure 4 shows the results of the transfer test, during which the diffuse stimuli were presented in combination with the white keylight. During that test, both stimuli augmented responding to $\mathrm{W}$, but only the augmentation produced by the otherwise untreated $\mathrm{Y}$ facilitator was reliable $[T(15)=20, p<.02]$. That stimulus also showed reliably greater augmentation than did the diffuse $\mathrm{X}$ stimulus treated otherwise as an inhibitor $[T(14)=0, p<.01]$.

These results suggest that concurrent inhibitory training interferes with the development of conditioned facilitation. As in Experiment 1, that interference was evident during both acquisition and transfer. In the case of facili-

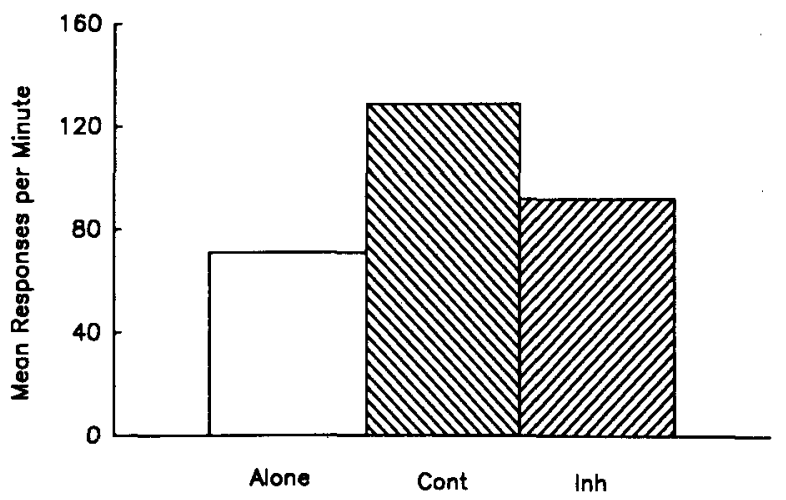

Figure 4. Transfer of facilitation in Experiment 2. Responding is shown to a keylight transer stimulus when it was presented alone and when it was in combination with the inhibitory and control facilitators. tation, the effect in transfer is especially important because it provides an assessment of the modulatory effects on a comparable target stimulus.

\section{EXPERIMENT 3}

This experiment had two intentions. First, it sought to compare directly the effects of facilatory and excitatory training on the development of inhibitory properties by a stimulus. Previous results (e.g., Rescorla, 1991) have shown that concurrent excitatory training enhances the development of a stimulus as an inhibitory modulator. Experiment 1 of the present report suggests by contrast that concurrent facilitory training interferes with that development. It would be of interest to compare these two effects within the same experiment. This is particularly true because there are still occasional concerns that facilitation might simply reflect the conditioning of excitation (e.g., Brandon \& Wagner, 1991). Observing opposite effects of facilitory and excitatory training could help to allay such concerns. Second, Experiments 1 and $2 \mathrm{em}-$ ployed diffuse stimuli as the modulators. It would be of interest to extend these observations to the case of localized visual modulators.

Consequently, in the present experiment, three different localized keylights were trained as inhibitors of a common excitatory target. One of those inhibitors received concurrent excitatory training, one received concurrent facilitory training, and one received no additional training.

\section{Method}

Subjects and Apparatus. The subjects were 16 experienced female pigeons with histories similar to those of the pigeons in $\mathrm{Ex}^{-}$ periment 1 and maintained in a similar fashion. The apparatus was that from Experiment 1. In addition to the four circular stimuli, two colored forms could be presented, a purple vertical bar $.5 \mathrm{~cm}$ in width and $2.5 \mathrm{~cm}$ high, and an inverted green equilateral triangle, $2.2 \mathrm{~cm}$ on a side.

Procedure. To ensure low levels of responding to all stimuli, the animals initially received nonreinforced exposure over 3 days. Each day contained twelve 5 -sec presentations of the $45^{\circ}$ pattern, the $135^{\circ}$ pattern, the pinwheel, the purple bar, and the green triangle.

On each of the next 8 days, the animals received conditioned inhibition training. Each day contained 12 reinforced presentations of the purple bar and 6 nonreinforced presentations of the bar immediately preceded by each of the three patterns. In addition, one of the patterns $(Y)$ was presented 12 times and followed immediately by food. Another pattern $(X)$ was trained in a facilitation procedure with the green triangle. This involved 12 reinforced presentations of the pattern in compound with the triangle, and 24 nonreinforced presentations of the triangle alone. On compound trials, the pattern was presented for $5 \mathrm{sec}$ and terminated $5 \mathrm{sec}$ prior to the onset of the target. This procedure was used in order to reduce the excitatory conditioning of the pattern that would have resulted without a gap between the stimuli. This temporal relation has routinely been used successfully to train patterns as modulators (see, e.g., Rescorla, 1985). The identity of the pattern that was excitatory (Y), facilitory (X), or not presented (Z) was counterbalanced across animals.

\section{Results and Discussion}

Figure 5 shows the results of the modulatory training. The filled symbols show responding to the vertical bar 
when it was presented alone and when it was preceded by each of the inhibitors. There were no substantial differences as a function of identity of the modulatory pattern; consequently the data are combined across stimulus identity but separated according to the treatment of the inhibitor elsewhere. It is clear that over the course of training all inhibitors came to reduce responding to the bar. On the final 2 days of training, all reliably suppressed responding $[T \mathrm{~s}(15)=0, p \mathrm{~s}<.01]$. However, the inhibitors were differentially effective, with separate excitatory training $(\mathrm{Y})$ producing the strongest inhibitor and separate facilitory training $(\mathrm{X})$ producing the weakest. On the last 2 days of training, each of these was reliably different from the nonpresented $(Z)$ inhibitor $[T s(14)<18$, $p s<.05]$, as well as from each other $[T(14)=15.5$, $p<.05$ ].

The open symbols show the results of responding to the green triangle. Although it was small, reliable facilitation of responding to the triangle was obtained from the modulator. On the final 2 days of training $[T(14)=7, p<$ .011 . The relatively weak effect of facilitation may well reflect the fact that the facilitator was concurrently being trained as an inhibitor, using a procedure with a shorter time gap before the target.

The levels of responding during the modulators themselves reflected their differential treatments. For instance, on the final day of training, the mean numbers of responses per minute were $112.4,9.0$, and 4.9 during the excitor, facilitator, and nonpresented inhibitors, respectively. The low level of responding to the facilitator is consistent with prior results from using localized visual stimuli separated in time from the target.

These results extend the demonstration of an adverse effect of facilitation training on inhibition to the case of localized visual modulators. They also verify that concurrent excitatory and facilitory training have quite different effects on inhibitory learning about a stimulus. Consequently, they provide additional support for the proposition that ex-

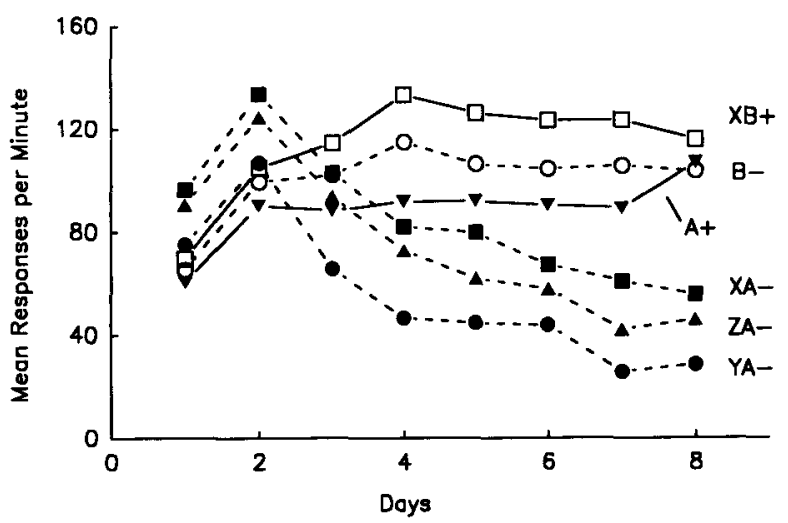

Figure 5. Acquisition of inhibition in Experiment 3. The filled symbols show responding during the excitatory $A$ stimulus when it was presented alone and when it was in combination with the localized inhibitors that were otherwise made excitatory $(Y)$, facilitory $(X)$, or not presented (Z). The open symbols show responding to $B$ when it was presented alone and when it was preceded by $\mathbf{X}$. citation and facilitation are functionally different types of learning.

\section{EXPERIMENT 4}

This experiment sought to combine the procedures of the previous experiments, evaluating within the same animal the impact of facilitation training on inhibition as well as the impact of inhibition training on facilitation. For this purpose, each animal was trained in three modulatory discriminations: facilitation $(\mathrm{A}-, \mathrm{XA}+)$, inhibition $(\mathrm{B}+$, $\mathrm{YB}-)$, and ambiguous cue $(\mathrm{C}-, \mathrm{ZC}+, \mathrm{D}+, \mathrm{ZD}-)$. In this procedure, $\mathrm{X}$ and $\mathrm{Y}$ are trained as simple modulators and $\mathrm{Z}$ is trained as both an inhibitor and a facilitator, using different targets. One can then compare the rate at which $\mathrm{Z}$ comes to inhibit $\mathrm{D}$ with the rate at which $\mathrm{Y}$ comes to inhibit $\mathrm{B}$ in order to assess the negative impact of the $\mathrm{C}-, \mathrm{ZC}+$ training on the development of inhibition to $\mathrm{Z}$. Similarly one can compare the rate at which $\mathrm{Z}$ comes to facilitate $\mathrm{C}$ with that at which $\mathrm{X}$ comes to facilitate $\mathrm{A}$ in order to assess the negative impact of $\mathrm{D}+, \mathrm{ZD}-$ on the development of facilitation to $\mathrm{Z}$.

\section{Method}

Subjects and Apparatus. The subjects were 16 experienced female pigeons with histories similar to those of the pigeons in Experiment 1 and maintained in a similar fashion. The apparatus was that of Experiment 1 . In addition to the stimuli previously used, two new colored forms were added: a brown rhombus, $2.2 \mathrm{~cm}$ long and $1.4 \mathrm{~cm}$ high, and a blue five-pointed star whose points were located on the circumference of an invisible $2.2-\mathrm{cm}$ circle. The $45^{\circ}$, $135^{\circ}$, and pinwheel patterns served in the roles of modulators, counterbalanced across animals; the purple rod, green triangle, brown rhombus, and blue star served as targets.

Procedure. Initially the animals received 4 days of preexposure to the stimuli. Each day contained 12 presentations of each of the seven stimuli. On the next 8 days, they received pretraining of the targets of modulation. On each of these days, they received 12 presentations of each of the colored forms. Two of the forms that would later serve as B and D terminated in grain; the two that would later serve as $A$ and $C$ terminated without grain.

On the next 24 days, the animals received modulation training. Each day contained eight trials of each of eight types: A-, XA +, $\mathrm{B}+, \mathrm{YB}-, \mathrm{C}-, \mathrm{ZC}+, \mathrm{D}+, \mathrm{ZD}-$. All stimuli were $5 \mathrm{sec}$ long, and on compound trials the pattern $(X, Y$, or $Z$ ) terminated $5 \mathrm{sec}$ prior to the onset of the colored form.

On the next day, the animals received a test. This test began with a half-session of modulation training and continued with another half session during which $X$ and $Y$ were interchanged with $Z$. This test allows an evaluation of the degree to which an ambiguous cue can modulate the targets of simple facilitators and inhibitors as well as the degree to which those simple modulators can affect the targets of an ambiguous cue.

\section{Results and Discussion}

Figures 6 and 7 show the results of modulation training in blocks of 4 days. In Figure 6 are plotted the data from the simple facilitation paradigm and from the facilitation portion of the ambiguous cue procedure. Figure 7 shows the comparable data from the inhibition treatments.

The solid symbols of Figure 6 show the results of simple facilitation training $(\mathrm{A}-, \mathrm{XA}+)$; the open symbols show those from the ambiguous cue procedure $(\mathrm{C}-$, 


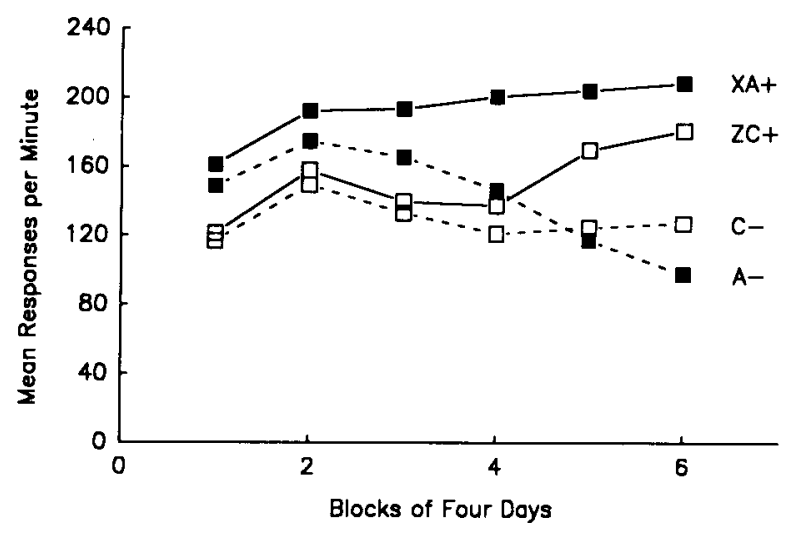

Figure 6. Acquisition of facilitation in Experiment 4. The filled symbols show responding in the simple facilitation paradigm to the target $A$ when it was presented alone and when it was preceded by $X$. The open symbols show responding in the ambiguous cue paradigm to the target $C$ when it was presented alone and when it was preceded by the ambiguous cue $\mathbf{Z}$.

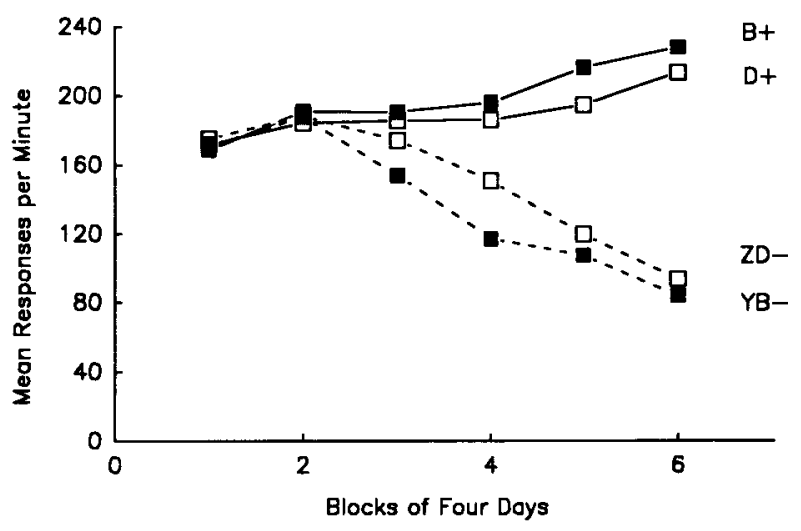

Figure 7. Acquisition of inhibition in Experiment 4. The filled symbols show responding in the simple inhibition paradigm to the target $B$ when it was presented alone and when it was preceded by $Y$. The open symbols show responding in the ambiguous cue paradigm to the target $D$ when it was presented alone and when it was preceded by the ambiguous cue $Z$.

$\mathrm{ZC}+$ ). The discrimination was successfully acquired in both cases. On the last block of training, a significant difference was observed in responding to the modulated and nonmodulated targets in both the simple facilitation $[T(14)=3, p<.01]$ and the ambiguous cue procedure $[T(15)=4, p<.01]$. However, the difference between responding to XA and $\mathrm{A}$ was greater than the difference in responding between $\mathrm{ZC}$ and $\mathrm{C}$. On the final block of training, that discrimination was reliably superior $[T(15)=$ $16, p<.01]$. As in Experiment 2, facilitation developed better if the modulator was not also getting conditioned inhibition training (as it does in the ambiguous cue paradigm).

The solid symbols of Figure 7 show the results of simple inhibition training $(\mathrm{B}+, \mathrm{YB}-)$; the open symbols show those from the ambiguous cue procedure $(D+$, $\mathrm{ZD}-$ ). In both procedures, the modulator came to inhibit responding to the target. On the final block of training, the difference was reliable both for simple inhibitory training $[T(15)=1, p<.01]$ and for the ambiguous cue procedure $[T(15)=2, p<.01]$. Of more interest, the difference between response rates to $B$ and $Y B$ was greater than that between responding to $\mathrm{D}$ and $\mathrm{ZD}$. On the final block of trials $[T(16)=27, p<.05]$. As in Experiment 2 , inhibition developed better if the modulator was not also getting facilitation training (as it does in the ambiguous cue paradigm).

Figure 8 shows the results of the transfer test. That figure displays responding to each of the four target stimuli under three different circumstances: when presented alone (first bar in each set), when accompanied by its training modulator (second bar), and when accompanied by the modulator from the other type of discrimination (third bar). The two sets of bars to the left show the results of testing with the target of simple facilitation and inhibition. It is clear that each of these A and B targets continued to be modulated by its original modulator, $X$ and $Y$, respectively. Of more interest, they were also modulated by the ambiguous cue, $\mathrm{Z}$. Responding on $\mathrm{A}$ was enhanced both by $\mathrm{X}[T(15)=5, p<.01]$ and by $\mathrm{Z}$ $[T(13)=9.5, p<.01]$. Responding on $B$ was depressed both by $\mathrm{Y}[T(13)=3, p<.01]$ and by $\mathrm{Z}[T(14)=1$, $p<.01]$.

A similar pattern was observed with the targets of the ambiguous cue training, C and D, as shown in the third and fourth set of bars in Figure 8. Responding during these targets was appropriately modulated by their original $Z$ as well as by the transfer $X$ and $Y$ stimuli. Responding during the target of $Z$ 's facilitation, $\mathrm{C}$, was enhanced both by $\mathrm{Z}[T(15)=5, p<.01]$ and by $\mathrm{X}[T(14)=20$, $p<.05]$. On the other hand, responding during the target of Z's inhibition, D, was depressed by both $\mathrm{Z}$ $[T(14)=4, p<.01]$ and by $Y[T(14)=5, p<.01]$.

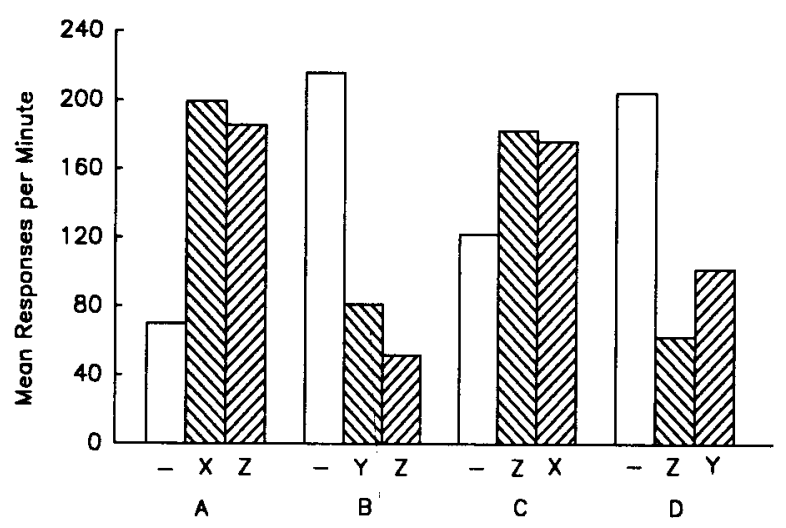

Figure 8. Transfer test of inhibition and facilitation in Experiment 4. Responding is shown to the targets of the simple facilitation (A) and inhibition (B) paradigms as well as to the facilitation (C) and inhibition (D) targets of the ambiguous cue paradigm. In each case, the target was presented alone (open bar), preceded by its original modulator (middle bar), and preceded by the transfer modulator from the other paradigm (right bar). 
The transfer of the simple facilitator and inhibitor to the targets of an ambiguous cue paradigm confirms earlier findings. When presented together with a transfer target, the facilitator promoted and the inhibitor interfered with responding. Not surprisingly, stimuli trained in an ambiguous cue paradigm served as appropriate targets for simple modulators.

The successful transfer of the ambiguous cue to the former targets of inhibition and facilitation is of somewhat more interest. Since the ambiguous cue has been trained to augment some stimuli and depress others, this raises the question of how the ambiguous cue appropriately augments (rather than depresses) the former target of a facilitator and appropriately depresses (rather than augments) the former target of an inhibitor. These results suggest the possibility that some of the information about the direction of modulation is encoded in the target. This must in some sense be true for solution of the ambiguous cue discrimination itself, since $\mathrm{Z}$ augments $\mathrm{C}$ and depresses $D$. But the transfer results suggest that it may also be true of the targets of simple modulators. This encoding within the target may be as simple as being dependent on its current level of excitation, or it may involve more complex processes.

\section{GENERAL DISCUSSION}

These experiments suggest that concurrent training with either facilitation or inhibition has an adverse effect on the development of the other. These results are consistent with the view that inhibition and facilitation are functional opposites of one another. Moreover, they suggest that excitation involves a different kind of learning, one of whose consequences is to promote both kinds of modulatory learning.

These results are consistent with previous results from performance: facilitators and inhibitors seem to transfer to other targets and to combine in a subtractive way when they transfer. Indeed, the previous observations of successful transfer virtually ensure the kind of interference observed in the present experiments.

At the same time, it is important to note that the interference effect observed here was relatively small. Furthermore, all of the procedures eventually resulted in successful solutions despite interference effects. Indeed, even the rather complex ambiguous cue procedure of Experiment 4 eventually resulted in contingency-appropriate performance. That successful solution agrees with the observation that in this procedure transfer across stimuli is far from complete (e.g., Rescorla, 1985, 1991). The result is that the ambiguous cue can serve as an inhibitor for one target and a facilitator for another. That is, the action of a modulator can be highly target specific. That observation conforms better to a hierarchical interpretation of modulation (e.g., Holland, 1985) than to one in terms of action on the US representation (e.g., Rescorla, 1985).
These results are different from those found with the use of an instrumental procedure by Holland (1991) in that a measurable interference effect was obtained. But they are like those of Holland in that a modulator can come to have opposite effects on different target stimuli. Moreover, they share with Holland's the transfer of the modulator in an appropriate way between the ambiguous cue and simple modulatory tasks.

One might well argue that the present results suggest a modulation process that is primarily target specific. For instance, a modulator might act by increasing the effectiveness of the association between its target and the US (e.g., Holland, 1985). Within such a framework, the interference observed here, and the transfer observed elsewhere, must represent generalization across targets or across modulators. Although the stimuli selected for the present experiments were intended to minimize such transfer, there is no way to guarantee that this intention was fulfilled.

The possibility of generalization across targets also suggests that a good portion of modulation and its transfer might be due to learning about stimulus compounds. For instance Pearce (e.g., Wilson \& Pearce, 1990) has argued that the XA compound in an XA +, A - facilitation procedure is treated as a configural unit that gains its own excitatory strength. On that account, there is no separate modulatory process, but only simple excitation and inhibition to various compounds. As Pearce has noted, a good many of the available modulation results can be accommodated within such a framework. In particular, the oppositeness of the products of inhibitory and facilitatory training would follow naturally. However, some of the results from comparing facilitation, excitation, and inhibition seem difficult for that approach to explain. For instance, it does not deal naturally with the observation that excitatory training promotes the development of inhibition, as seen in Experiment 3 and as more extensively studied by Rescorla (1991). Nor does it anticipate that facilitation and excitation training would have opposite effects on the development of an inhibitor.

Nevertheless, as the data on modulation become increasingly complex, it seems increasingly likely that there are multiple determinants. These may well include action on the CS (as suggested by generalization models such as that described by Pearce), action on the US (as suggested by threshold-shifting models such as that described by Rescorla), and action on the relation between the CS and US (as described by Holland).

\section{REFERENCES}

Brandon, S. E., \& Wagner, A. R. (1991). Modulation of a discrete Pavlovian conditioned reflex by a putative emotive Pavlovian conditioned stimulus. Journal of Experimental Psychology: Animal Behavior Processes, 17, 299-311.

Holland, P. C. (1983). "Occasion-setting" in Pavlovian feature positive discriminations. In M. L. Commons, R. J. Herrnstein, \& A. R. 
Wagner (Eds.), Quantitative analyses of behavior: Vol. 4. Discrimination processes (pp. 183-206). Cambridge, MA: Ballinger.

Holland, P. C. (1985). The nature of conditioned inhibition in serial and simultaneous feature negative discriminations. In R. R. Miller \& N. E. Spear (Eds.), Information processing in animals: Conditioned inhibition (pp. 267-297). Hillsdale, NJ: Eribaum.

Holland, P. C. (1991). Transfer of control in ambiguous discriminations. Journal of Experimental Psychology: Animal Behavior Processes, 17, 231-258.

Rescorla, R. A. (1985). Inhibition and facilitation. In R. R. Miller \& N. E. Spear (Eds.), Information processing in animals: Conditioned inhibition (pp. 299-326). Hillsdale, NJ: Erlbaum.

RESCORLA, R. A. (1986). Extinction of facilitation. Journal of Experimental Psychology: Animal Behavior Processes, 12, 16-24.
Rescorla, R. A. (1987). Facilitation and inhibition. Journal of Experimental Psychology: Animal Behavior Processes, 13, 250-259.

Rescorla, R. A. (1991). Separate reinforcement can enhance the effectiveness of modulators. Journal of Experimental Psychology: Animal Behavior Processes, 17, 259-269.

Wilson, P. N., \& PEARCE, J. M. (1990). Selective transfer of responding in conditional discriminations. Quarterly Journal of Experimental Psychology, 42B, 41-58.

(Manuscript received August 8, 1992; revision accepted for publication January 2, 1993.) 